\title{
ORIGAMI, une méthode organisation- centrée de modélisation multi-agent de systèmes complexes
}

\author{
Géraldine Abrami* - Sylvie Lardon** — Olivier Barreteau*** \\ Flavie Cernesson****
}

* Cirad, UR Green, TA 60/1, 73 rue Jean-François Breton

F-34398 Montpellier cedex 5,

geraldine.abrami@cirad.fr

** INRA-Engref POP'TER, UMR Métafort, 24, avenue des Landais

Domaine universitaire des Cézeaux, BP 90054, F-63171 Aubière cedex 9

*** Cemagref UR Usages, 361 rue JF Breton BP 5035

F-34033 Montpellier cedex

**** Cemagref / Engref UMR Tétis, 500 rue JF Breton BP 90054

F-34033 Montpellier cedex

RÉSUMÉ. Dans le champ de la gestion de ressources renouvelables, les acteurs (chercheurs,
experts, partie prenantes) doivent appréhender des systèmes complexes où des dynamiques
sociales et physiques s'inscrivent dans des niveaux d'organisation multiples. La modélisation
conceptuelle de ces systèmes permet de partager des points de vue et d'améliorer la
compréhension. Dans ce contexte, nous proposons d'utiliser un formalisme multi-agent
organisationnel nommé Agent-Groupe-Rôle accompagné d'une méthodologie graphique
basée sur le langage UML nommée ORIGAMI. Cette méthodologie a été développée sur la
base d'un modèle-jouet et testée lors d'une expérience de formation. Le recours à cette
méthodologie permet d'expliciter des hypothèses d'habitude implicites et de mieux rendre
compte des différents niveaux d'organisation d'un système et de leurs articulations.
ABSTRACT. In the field of renewable resources management, actors (researchers, experts,
stakeholders) are facing complex systems where social and physical dynamics take place
within various interwoven levels of organization. Conceptual modelling is a way to share
understandings and point of views of these various actors. In this context, we present an
approach based on a multi-agent formalism called Agent-Group-Role. In order to implement
this approach, we have developed a graphic methodology based on UML and called
ORIGAMI. This methodology was built extensively on the basis of a toy model and has been
tested during a training experiment. It has proven efficient in representing interwoven levels
of organisation and their articulations and in explicating usually hidden assumptions.
MOTS-CLÉS: modélisation multi-agent, méthodologie de conception, rôles, gestion de l'eau,
Drôme
KEYWORDS: agent-based modelling, design methodology, roles, water management, Drôme

Géomatique - 16/2006. CQFD, pages 
2 Géomatique - 16/2006. CQFD

\section{Introduction}

Le contexte dans lequel se place ce travail est celui de la conception coopérative de modèles destinés à l'accompagnement de projets de gestion de ressources naturelles communes. Les problématiques de gestion de ressources naturelles mettent en jeu des acteurs (individus ou organisations) multiples en interaction autour d'une ressource et amènent à considérer à la fois des dynamiques physiques et sociales, et ce à différentes échelles (parcelle, exploitation, territoire) et à différents niveaux organisationnels (communauté villageoise, politique régionale) ou temporels (constructions de règles de gestion, mise en œuvre de règles de gestion) (Heathcote 1998). Au-delà de l'approche systémique, le recours à la modélisation, et plus particulièrement à la modélisation multi-agent, est un moyen d'aborder ces systèmes complexes, et ce afin d'en améliorer la compréhension tout en offrant un support intégrateur des différents points de vue scientifiques et profanes permettant de médier les discussions entre scientifiques et/ou entre parties prenantes. (Bousquet, Barreteau et al., 2002 ; Bousquet and Le Page 2004).

Le développement du modèle multi-agent d'un système est un processus cyclique dans lequel se succèdent des phases de recueil de données, de modélisation conceptuelle, d'implémentation informatique et de simulation (Bousquet 2001). La phase de modélisation conceptuelle permet de clarifier les frontières du système, de mieux formuler les questions, de révéler les hypothèses sous-jacentes et les confusions terminologiques, et d'identifier les trous d'information. Cette phase implique généralement la collaboration entre un modélisateur et des individus porteurs de connaissances sur le systèmes. Ainsi, le modèle conceptuel, au delà d'un objectif et d'un produit, est un objet central médiant la communication entre les acteurs (chercheurs, experts, éventuellement parties prenantes) impliqués dans le processus de modélisation et supportant le partage et la mise en commun de leurs points de vue (Heemskerk, Wilson et al., 2003). Il est donc important de disposer d'outils de représentation à la fois compréhensibles par tous afin de pouvoir servir de support de communication, et suffisamment formalisés pour fournir la précision nécessaire aux spécifications d'un modèle informatique.

L'objet de cet article est, dans ce contexte, en s'appuyant sur les apports existants de l'approche systémique, de présenter une méthode de construction de modèles conceptuels adaptée à la nature complexe des systèmes en jeu dans la gestion de ressources naturelles communes. La méthode exposée utilise les bases méthodologiques du langage UML et de l'approche objet. Son originalité tient dans l'utilisation de concepts issus du formalisme Agent-Groupe-Rôle (AGR), qui permet de mener une analyse organisationnelle des systèmes cibles.

UML (Unified Modelling Language) est un langage semi-formel aujourd'hui standard permettant d'exprimer ou d'élaborer des modèles orientés objet, indépendamment de tout langage de programmation. UML propose un support de notation graphique constitué de plusieurs types de diagrammes permettant 
d'exprimer différentes vues sur un modèle ${ }^{1}$. Parce qu'il a été conçu pour représenter un système informatique à travers la description de ses entités, de leurs activités et de leurs interactions, UML s'est révélé un support adapté à la conceptualisation de modèles systémiques (Roux-Rouquie, Caritey et al., 2004). De plus son temps d'apprentissage est suffisamment réduit pour pouvoir rapidement être utilisé comme support de modélisation conjointe de systèmes de gestion de ressources communes (Le Page and Bommel 2005).

Les systèmes multi-agents (SMA) trouvent leur pertinence dans l'accompagnement à la décision collective sur des systèmes complexes grâce à leur capacité à représenter par des règles simples les actions et interactions d'entités hétérogènes dans un environnement partagé. En complément à cette approche individu-centrée, un courant de la recherche informatique sur les SMA promeut une approche des systèmes multi-agent centrée non plus sur l'activité des agents mais sur la structuration des systèmes et inspirée par des analogies issues de la sociologie des organisations, et a débouché sur un formalisme ${ }^{2}$ appelé Agent-Groupe-Rôle (AGR) (Ferber and Gutknecht 1998). Le recours à ce nouveau type de formalisme nous a semblé nécessaire dans la mesure où l'analyse systémique, classiquement à la base de la conceptualisation d'un modèle SMA, et consistant à décrire les entités du systèmes et leurs interactions bute parfois sur des écueils liés à la nature intrinsèquement organisationnelle des systèmes considérés :

- Les entités d'un système y occupent en général des fonctions multiples, fonctions pouvant être communes à plusieurs entités du systèmes : une même entité peut porter différentes «casquettes » (e.g. maire et conseiller régional), elle peut intervenir dans plusieurs niveaux du système (e.g. intervenir dans l'élaboration et dans la mise en œuvre d'un projet), ou bien elle peut posséder types d'activités (e.g. agriculteur et bâtisseur) ou facette (e.g. ressource et capital). Comment alors dissocier une entité de ses fonctions, un objet de ses usages?

- L'analyse d'un tel système implique généralement plusieurs échelles structurelles (e.g. la parcelle, l'exploitation, le conseil municipal), plusieurs points de vue disciplinaires (e.g. le système de production, le processus de décision, l'organisation du marché), plusieurs temporalités (e.g. l'élaboration, la mise en œuvre, l'impact) conduisant à l'identification de sous-systèmes entremêlés et interdépendants. Comment alors décrire et concilier ces différents sous-systèmes?

Le formalisme AGR, qui intègre les concepts de rôle et de structure organisationnelle au cœur de la description d'un SMA nous est apparu comme

\footnotetext{
${ }^{1}$ Pour plus d'informations, on pourra se référer au site officiel de l'Object Management Group, qui définit les standards d'UML http://www.omg.org/, et plus particulièrement au site officiel d'UML http://www.uml.org.

${ }^{2}$ Un formalisme est défini comme un système symbolique définissant des règles pour manipuler des objets sans avoir besoin de se référer au contenu des objets ni au contexte (Heylighen, F. (1999). "Advantages and limitations of formal expression." Foundations of Science 4(1): 25-56).
}

Mise en forme : Puces et numéros 
présentant des caractéristiques susceptibles de répondre à ces questions de manière générique. Ce formalisme issu d'analogies sociétale a été développé dans un objectif générique de renforcement et d'enrichissement des approches de conception de SMA. Il nous a donc fallu dans un premier temps adapter sa sémantique naturelle à notre domaine d'application, la modélisation multi-agent, et plus particulièrement la modélisation multi-agent pour la gestion de ressources communes, qui constitue une branche bien particulière des SMA (Drogoul, Vanbergue et al., 2003). Il nous a fallu ensuite construire une méthode permettant de construire le modèle AGR conceptuel d'un système et de le communiquer. La méthode ORIGAMI (Original Roles Identification, Groups and Agents Modelling Itinerary) s'appuie sur le formalisme UML et permet une analyse organisationnelle des systèmes. Elle s'est bâtie au fil des expérimentations sur un modèle-jouet de la gestion quantitative de l'eau dans la basse-vallée de la Drôme, dans le Sud de la France (Abrami, Barreteau et al., 2003). C'est la description de cette méthode qui fait l'objet de cet article.

L'article débute par une section matériel et méthodes où, après avoir décrit le formalisme AGR, on présente de manière globale la méthode ORIGAMI, et enfin on expose le cas d'étude qui sert de base à notre modèle-jouet. Les différents éléments de la méthodologie ORIGAMI sont ensuite présentés en détail au long des deux sections suivantes : l'exemple du modèle jouet est développé d'abord sur ses aspects statiques, puis sur ses aspects dynamiques. Enfin, l'approche est discutée sur sa capacité à appréhender la complexité d'un système ainsi que sur la base de l'expérience de formation où elle a été présentée.

\section{Matériel et méthodes}

Le but de cette section est de présenter l'outillage théorique et méthodologique utilisé dans ce travail : le formalisme AGR qui définit les concepts de base de notre approche, la méthode ORIGAMI qui a été développée afin de pouvoir utiliser ces concepts dans des situations de modélisation, et enfin le cas d'application que l'on utilise pour construire un modèle-jouet.

\subsection{Le formalisme Agent-Groupe-Rôle}

Le formalisme Agent-Group-Rôle de (Ferber and Gutknecht 1998) est ainsi défini :

- Un agent est défini comme une entité active communicante, qui prend en charge des rôles dans des groupes. Un agent devient membre d'un groupe en venant prendre en charge un rôle défini par ce groupe. Il peut alors prendre en charge simultanément d'autres rôles de ce groupe, ou bien des rôles définis dans d'autres groupes, dont il devient alors aussi membre. Aucune hypothèse n'est faite sur l'architecture des agents. 
- Un groupe est constitué d'un ensemble d'agents interagissant à travers leurs rôles. A un niveau abstrait, il définit un ensemble de rôles et de relations entre ces rôles réalisant une fonction globale. Un groupe s'instancie alors par la prise en charge de ses rôles par des agents, mais tous les rôles qui y sont peuvent ne pas être pris en charge, et un même rôle peut être instancié plusieurs fois sur différents agents. Le groupe constitue donc une structure de description dotée d'un très fort niveau d'abstraction et capable de générer une grande diversité de réalisations. Enfin, les groupes sont opaques les uns envers les autres: des agents ne peuvent interagir que s'ils appartiennent à un même groupe.

- Un rôle est défini comme la représentation abstraite de la fonction d'un agent dans un groupe. Les rôles sont définis dans des groupes et encapsulent la manière Mise en forme : Puces et dont un agent devrait agir au sein du groupe.

Les concepts de rôle, de groupe et d'agent fournissent des abstractions organisationnelles adaptées à surmonter les écueils récurrents à la conceptualisation d'un modèle multi-agent d'un système de gestion de ressources communes relevés dans l'introduction. Avant de décrire les correspondances possibles entre ces abstractions et des objets d'un système, nous introduisons le concept de niveau d'organisation. Nous utilisons le terme niveau d'organisation pour désigner une unité de description organisationnelle complémentaire aux unités de description individuelles telles que les acteurs ou les objets. Cette terminologie de niveau d'organisation recouvre la désignation des différents sous-systèmes pouvant émerger de l'analyse d'un système selon le prisme de différents points de vue disciplinaires, différentes échelles ou différentes temporalités (systèmes de production, marchés, processus biophysiques d'une parcelle, systèmes de mise en œuvre d'une règle de gestion collective...). Nous définissons un niveau d'organisation comme le point de vue d'un modélisateur sur un ensemble d'entités du système accomplissant une fonction globale donnée dans une unité structurelle cohérente.

Les abstractions AGR peuvent alors être interprétées ainsi :

- Le groupe représente un niveau d'organisation, soit un sous-système constitué Mise en forme: Puces et d'entités interagissant autour d'une fonction donnée et dans une unité spationuméros temporelle cohérente. A un niveau abstrait, un groupe est composé de rôles qui représentent l'ensemble des fonctions et relations qui caractérisent ce niveau d'organisation et qui peuvent être décrites indépendamment des composantes du système qui les implémentent.

- Un rôle représente la contribution d'une entité à un niveau d'organisation. Un rôle décrit les activités de l'entité dans le niveau d'organisation, et définit ce qui peut être perçu ou contrôlé de l'entité depuis le niveau d'organisation.

- Un agent définit les caractères internes et objectifs d'une entité (alors qu'un rôle traduit l'implication fonctionnelle des agents au sein des organisations). La personnalité d'une entité se définit dans ses méthodes de choix des rôles qu'elle pourra prendre en charge, ainsi que par l'ensemble des rôles qui lui sont accessibles. 
Ainsi, le formalisme AGR fournit des briques de construction fonctionnelles et organisationnelles permettant de conceptualiser des unités de description d'un système qui viennent enrichir les concepts d'entité et d'interaction propres aux SMA.

\subsection{La méthode ORIGAMI}

ORIGAMI se base sur l'utilisation de diagrammes UML pour construire des représentations organisationnelles d'un système basées sur le formalisme AGR. ORIGAMI permet donc de structurer l'analyse fonctionnelle et systémique d'un système à travers l'identification de ses unités structurelles, des fonctions que l'on souhaite en décrire, et des entités le constituant. ORIGAMI fournit des séries de diagrammes permettant d'une part une description de la structure d'un système, et d'autre part une description de ses dynamiques.

La description statique d'un système s'y fait suivant 4 étapes possibles :

- Etape 1. Identification des niveaux d'organisation du système : il faut pour cela identifier quelles sont les fonctions globales et les unités structurelles du système que l'on souhaite représenter. Un tableau structures/fonctions peut alors être utilisé pour croiser fonctions et unités structurelles et en tirer les niveaux d'organisation. Un diagramme des classes présentant les relations fonctionnelles entre ces niveaux d'organisation, appelé diagramme fonctionnel global, permet alors de visualiser globalement les articulations entre ces niveaux d'organisation.

- Etape 2. Identification des entités individuelles du système : il convient simplement à cette étape d'établir la liste des entités individuelles qui interviennent dans les différents niveaux d'organisation identifiés lors de l'étape précédente.

- Etape 3. Identification des fonctions des entités au sein des niveaux d'organisation: cette étape revient à identifier les rôles de la représentation AGR du système. On utilise pour cela des diagrammes appelés diagrammes swimlane, introduits par (Parunak and Odell 2002). Ces diagrammes swimlane sont en fait des diagrammes de classe UML où les niveaux d'organisation apparaissent en colonnes, les agents en ligne, et les rôles à la croisée des agents et des niveaux d'organisation. La description des rôles présents dans les différents niveaux d'organisation peut alors être affinée par l'utilisation de diagramme de classes décrivant comment certains de ces rôles peuvent être spécialisés.

- Etape 4. Identification des liens structurels entre les entités : les liens structurels (permanents et intrinsèques) entre les entités d'un système sous-tendent les liens fonctionnels établis à travers leurs rôles. Le diagramme structurel est un diagramme des classes UML décrivant uniquement ces liens structurels et sur lequel il est possible de spécifier les contraintes que ces liens structurels font porter sur la prise en charge de rôles. 
La description des dynamiques d'un système peut alors se faire au travers de 3 types de diagrammes :

- des diagrammes d'activité pour décrire le déroulement des processus constituant la dynamique du système. Selon la portion de système sur laquelle portent ces diagrammes d'activité, différents niveaux de détail peuvent être représentés : des diagrammes d'activité globaux permettent de décrire l'articulation entre les niveaux d'organisation lors du déroulement d'un processus; des diagrammes d'activité organisationnels permettent de décrire la manière dont ces processus se déroulent au sein d'un niveau d'organisation; enfin, des diagrammes d'activité individuels permettent plus classiquement de décrire des pratiques ou des stratégies mises en œuvre par des agents ou des rôles ;

- des diagrammes de séquence pour décrire le séquencement des interactions. Là encore, selon le niveau de détail choisi, ces diagrammes peuvent se concentrer sur le séquencement des dynamiques intra et inter niveau d'organisation, ou bien sur le séquencement des dynamiques organisationnelles (prise en charge et abandon de rôles) ;

- des diagrammes d'état-transition pour décrire le cycle de vie des entités. Ces diagrammes peuvent être utilisés pour décrire les conditions de prise en charge et la succession des rôles pour un agent, ou bien pour décrire le cycle des états parcourus par un agent ou un rôle.

\subsection{Un modèle-jouet de gestion de l'eau dans un bassin versant irrigué}

La modélisation AGR d'un système avec la méthode ORIGAMI a été mise en œuvre sur une étude de cas de gestion de ressource renouvelable commune : la gestion collective de l'eau d'irrigation dans la basse-vallée de la Drôme, dans le Sud de la France.

A partir d'une étude de cas sur la gestion collective de l'eau d'irrigation dans la vallée de la Drôme (Zanker 1999), ainsi que d'un modèle SMA classique de cette situation (Barreteau, Garin et al., 2003), nous avons construit un exemple simplifié afin de tester la structure AGR dans sa capacité à représenter des niveaux d'organisation superposés ou imbriqués en interaction, ainsi que leurs relations aux individus.

Nous considérons l'usage agricole de l'eau dans une portion de bassin versant où coexistent trois systèmes irrigués ainsi que des pompes individuelles. Les agriculteurs cultivent du maïs dans plusieurs parcelles. Chaque parcelle est irriguée par un accès à l'un des systèmes irrigués ou bien par une pompe, mais un même agriculteur peut avoir des parcelles présentant différents accès à l'eau.

Les systèmes irrigués possèdent un accès direct à la rivière et répartissent leur eau entre leurs membres. Leur président, qui peut être un agriculteur, est un intermédiaire entre les membres du système et les niveaux de décision supérieurs. 
En cas de sécheresse, il est tenu d'adapter l'organisation interne du périmètre aux limitations administratives. L'irrigation individuelle est peu organisée et peut être soumise à des jours d'interdiction.

\section{Application de la méthode ORIGAMI : description de la structure du système}

La méthode ORIGAMI est à présent exposée en détail au travers de son utilisation sur le cas du modèle-jouet. On commence par s'intéresser aux aspects statiques. L'objectif de cette phase du processus de modélisation est d'identifier et de spécifier les différents composants du modèle ainsi que la manière dont ils sont reliés.

\subsection{Identification des niveaux d'organisation}

Cette première étape a pour but d'identifier les niveaux d'organisation du système et de nommer les groupes correspondants. Un niveau d'organisation étant défini comme un point de vue sur une fonction du système dans une unité structurelle pertinente, il faut donc lister les unités structurelles auxquelles on veut se placer, et les fonctions que l'on souhaite modéliser.

Les 4 unités structurelles sont définies par les différentes échelles hydrauliques et de gestion de la situation modélisée : le bassin, le périmètre irrigué, l'exploitation et la parcelle. On distingue par ailleurs 2 types de fonctions :

- la circulation de l'eau : propagation de débit de l'entrée du bassin à travers la rivière, puis de la prise d'eau du réseau aux bornes individuelles des irrigants, et enfin de la borne à la parcelle, où se déroule le bilan hydrique ;

- l'allocation de l'eau: mise en œuvre des règles de gestion individuelles et collectives par les différents acteurs.

Le tableau structure/fonction (tableau 1) présente les différents niveaux d'organisation identifiés dans la situation-jouet, ainsi que les noms groupes correspondants.

Les groupes issus des processus d'allocation de l'eau décrivent des fonctions d'usage de la ressource. Ils sont reliés par des relations d'ordre sociales, chaque groupe étant contraint par des règles issues d'un groupe d'un niveau plus agrégé. Les groupes issus des processus de circulation de l'eau décrivent des fonctions naturelles du système. Ils sont reliés par des relations d'ordre hydraulique, chaque groupe recevant de l'eau d'un groupe de niveau plus agrégé. Enfin, à chaque échelle, un groupe «social» contrôle les objets hydrauliques du groupe « hydraulique » correspondant (relation d'usage). 


\begin{tabular}{|c|l|l|}
\hline Structures/fonctions & \multicolumn{1}{|c|}{ Circulation de l'eau } & \multicolumn{1}{c|}{ Allocation de l'eau } \\
\hline Bassin & $\begin{array}{l}\text { Circulation de l'eau dans la } \\
\text { rivière (Basin) }\end{array}$ & $\begin{array}{l}\text { Observation de la rivière et } \\
\text { déclenchement des restrictions } \\
\text { par la CLE } \\
\text { (BasinManagement) }\end{array}$ \\
\hline Réseau & $\begin{array}{l}\text { Circulation de l'eau dans le } \\
\text { réseau (IrrigationNetwork) }\end{array}$ & $\begin{array}{l}\text { Exécution des restrictions, } \\
\text { répartition de l'eau entre les } \\
\text { irrigants } \\
\text { (IrrigationAssociation) }\end{array}$ \\
\hline Exploitation & $\begin{array}{l}|c| \\
\text { Allocation de l'eau entre les } \\
\text { parcelles (Farm) }\end{array}$ \\
\hline Parcelle & $\begin{array}{l}\text { Stockage de l'eau par le sol } \\
\text { et bilan hydrique (Crop) }\end{array}$ & \multicolumn{2}{|c}{} \\
\hline \multicolumn{2}{|c|}{}
\end{tabular}

Tableau 1. Niveaux d'organisation de la situation-jouet. Les groupes correspondants sont entre parenthèses

Ainsi, les groupes du modèle-jouet présentent les relations fonctionnelles résumées sur le diagramme figure 1 .

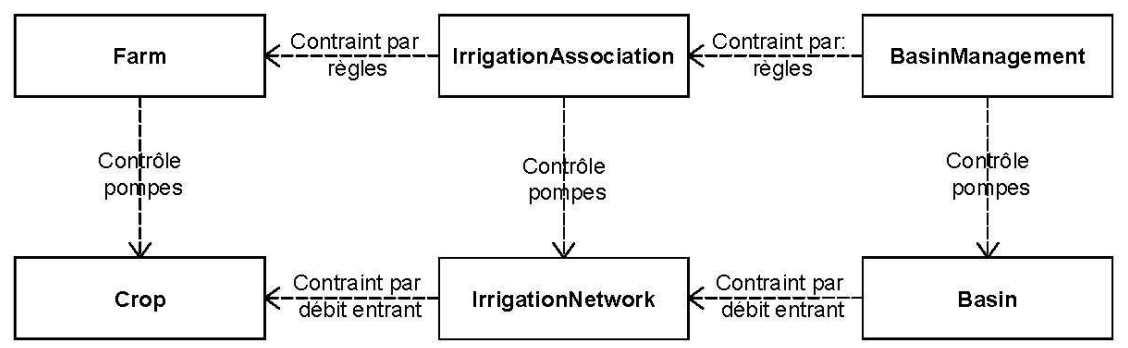

Figure 1. Diagramme fonctionnel global du modèle jouet. Ce diagramme n'est pas temporel, il représente les relations fonctionnelles entre les groupes

Une classification des niveaux d'organisation du système apparaît : structures de gestion d'une unité fonctionnelle d'un côté, unités fonctionnelles gérées de l'autre.

\subsection{Identification des entités individuelles}

L'objectif de cette étape est d'identifier quelles sont les entités individuelles (acteurs, organisations ou objets) intervenant dans les niveaux d'organisation 
identifiés lors de l'étape précédente. On entend par «individuel» un acteur, un objet ou une organisation que l'on considère se comporter comme une entité unique. Ces entités définissent alors les agents du modèle.

Entités sociales Ce sont les agriculteurs (agent Farmer) et la CLE (agent CLE). La CLE n'est pas un individu, mais puisque qu'on ne la considère qu'en tant qu'instance de gestion du bassin (on ne modélise pas la concertation), on peut la décrire comme une entité individuelle. L'association d'irrigants est par contre vue comme un groupe où ont lieu des communications, avec un président et des membres. Président et membre sont en fait des rôles du groupe association d'irrigants pris en charge par des agriculteurs. Ces agents sont essentiellement caractérisés par les séries de rôles qu'ils prendront en charge.

Entités physiques Ce sont les cultures (agents Plant et Maze), ainsi que les pompes (agents Pump), les parcelles (agent Plot), la rivière (agent River) et la conduite du réseau (agent Pipe). Les pompes des objets situés. Elles sont caractérisées par leur capacité et leur débit, et savent s'ouvrir et se fermer. Les TerminalPump sont les bornes des irrigants; elles desservent un ensemble de parcelles. Les HeadPump sont les prises du réseau; elles desservent une conduite. Une plante est caractérisée par divers coefficients culturaux. Les parcelles sont caractérisées par leur position, la profondeur leur sol, ainsi que par leur réserve en eau. Rivière et conduite sont caractérisées par leurs connections et leur position. Elles savent accéder à leur débit en un point donné. Enfin, on modélise le rafraîchissement des séries climatiques (débit entrant, pluie et évapotranspiration journalières) avec une entité Climate, qui est active, mais non spatiale (le système est suffisamment petit pour que le climat soit le même partout).

\subsection{Identification des fonctions des entités au sein des niveaux d'organisation}

L'outil utilisé lors de cette étape est le diagramme «swimlane ${ }^{3}$ », introduit par (Parunak and Odell 2002).

\subsubsection{Les diagrammes swimlane}

Le principe du diagramme « swimlane » est de croiser agents et groupes pour nommer les rôles que les agents doivent occuper dans les groupes. Les différentes classes du systèmes y sont organisées dans un tableau : chaque colonne du tableau représente un groupe et les différents rôles qu'il définit, alors que chaque ligne en représente un agent et les différents rôles qu'il est susceptible de prendre en charge.

\footnotetext{
${ }^{3}$ Des « swimlanes » (lignes de nage) ont déjà été introduits de manière standardisée dans les diagrammes d'activité UML (voir plus loin). Cependant c'est au type de diagramme introduit par Parunak et Odell que l'on se référera lorsque l'on parlera de diagramme swimlane dans ce document.
} 
On remplit le diagramme en répondant à la question «comment qualifier la participation de cette entité à ce niveau d'organisation?».

Une fois les rôles identifiés, on peut tracer leurs relations de communication. Les relations verticales représentent les communications entre les différents rôles définies dans un groupe : elles définissent la topologie des groupes et donnent une ébauche de leur fonctionnement; les relations horizontales représentent la circulation de l'information entre les différents rôles d'un même agent. La lecture verticale d'un diagramme swimlane permet d'accéder à la structure de chacun des niveaux d'organisation du système ; sa lecture horizontale permet d'en visualiser la superposition.

Les arités portées sur les liens horizontaux (pas d'arité signifie 1) sont très significatives : elles expriment des contraintes liées à la prise en charge de rôles multiples et reflètent les différentes configurations permises par la souplesse d'AGR. Ainsi, un lien 1 à 1 signifie que les 2 rôles doivent être simultanément pris en charge par le même agent, alors qu'un lien 1 à $0 . .^{*}$ signifie qu'un des rôles peut être pris en charge seul, mais que la prise en charge de l'autre dépend de celle du premier. Des contraintes peuvent aussi exister au niveau des groupes. On définit donc aussi des liens porteurs d'arités entre les groupes.

Quand les modèles sont complexes, on peut établir les diagrammes swimlane partiels. Le tracé d'un diagramme swimlane complet permet de revenir sur le découpage du système en niveaux d'organisation : les liens entre rôles peuvent faire apparaître des regroupements poussant à scinder un groupe en plusieurs, ou au contraire à faire de 2 groupes un seul (quand trop de liens horizontaux relient les 2 groupes). Les diagrammes swimlane sont des représentations synthétiques de la structure des systèmes. Ils constituent donc de bonnes bases pour affiner la dénomination et la répartition des différents éléments d'un système.

\subsubsection{Exemple du diagramme swimlane du modèle-jouet pour la circulation de l'eau}

Trois groupes sont concernées par la propagation du débit dans le système (voir tableau figure 1) : Basin, IrrigationNetwork et Crop. Les agents prenant part au processus sont les entités où passe de l'eau : Climate comme générateur de débit et de pluie, River et Pipe comme propagateurs de débit, Pump comme point de transfert de débit, et Plot comme réservoir d'eau. Groupes et entités constituent respectivement les colonnes et les lignes du diagramme swimlane figure 2.

Il faut maintenant déterminer quels sont les rôles définis dans chacune des groupes. Ces rôles apparaîtront sur le diagramme figure 2 comme étiquettes au croisement des agents et des groupes concernés. 


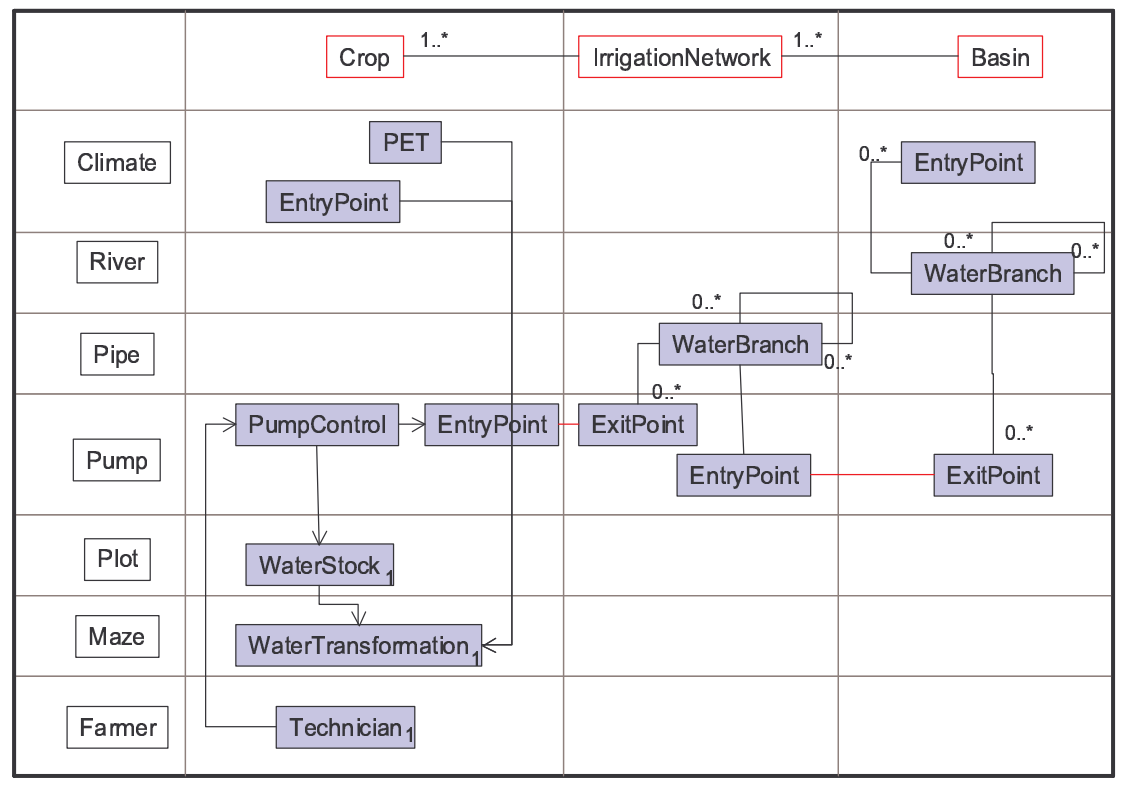

Figure 2. Diagramme swimlane des processus physique du modèle jouet

On représente la circulation de l'eau dans le système comme la propagation instantanée d'un flux à travers un système d'arcs et de nœuds, représentés par les rôles définis dans les différents groupes. Dans chacun des groupes, le débit se propage à partir d'un rôle EntryPoint (pris en charge par le climat qui donne le débit entrant pour le groupe Basin) à travers des rôles WaterBranch (pris en charge par les entités linéaires River et Pipe), jusqu'à des rôles ExitPoint (pris en charge par les pompes). Chaque point de sortie d'un groupe d'un niveau agrégé est le point d'entrée du groupe d'un niveau plus élémentaire. Dans les groupes Crop, le débit entrant est transformé en quantité d'eau et stocké par le rôle WaterStock, pris en charge par la parcelle. La quantité d'eau stockée est limitée par les caractéristiques de profondeur de sol de Plot.

Tous les jours, l'évapotranspiration, représentée par le rôle ETP de Climate agit et déclenche le bilan hydrique de WaterStock (consommation d'une partie du stock) et le rafraîchissement du rendement de la plante par WaterTransformation, en fonction de l'eau disponible et des coefficients culturaux de la plante.

Le processus de propagation de débit peut être déclenché par le rafraîchissement du débit entrant dans le système, mais aussi par l'ouverture ou la fermeture d'une pompe par un irrigant. On localise l'action d'irrigation dans les groupes Crop, où un agent Farmer prend en charge un rôle Technician. Le rôle Technician ne sait qu'actionner une pompe, la prise de décision est faite par un autre rôle de Farmer. 
Cette pompe est encapsulée dans les groupes Crop par un rôle PumpControl, qui modélise une interface permettant de contrôler la pompe. La pompe propage alors la demande de débit résultante à son rôle ExitPoint qui fait remonter cette demande jusqu'à la rivière par le circuit inverse de celui où le débit se propage.

\subsubsection{Exemple du diagramme swimlane pour l'allocation de l'eau au niveau de l'exploitation}

Le rôle Manager pris en charge par Farmer encapsule les règles de décision concernant la gestion de l'irrigation.

Manager a accès aux caractéristiques des différents objets de son exploitation à travers les rôles PlantObservation, PlotObservation et PumpObservation, qui fonctionnent à la manière d'instruments de mesure sur les objets par lesquels ils sont pris en charge. Ils donnent dans cette version du modèle une information exacte sur ces objets.

Quand le rôle Manager a pris les décisions concernant l'allocation de l'eau (établissement ou rafraîchissement d'un calendrier d'irrigation), le rôle Technician est chargé de mettre en œuvre ces décisions en programmant les actions d'irrigation. Le diagramme swimlane de ce processus est donnée figure 3.

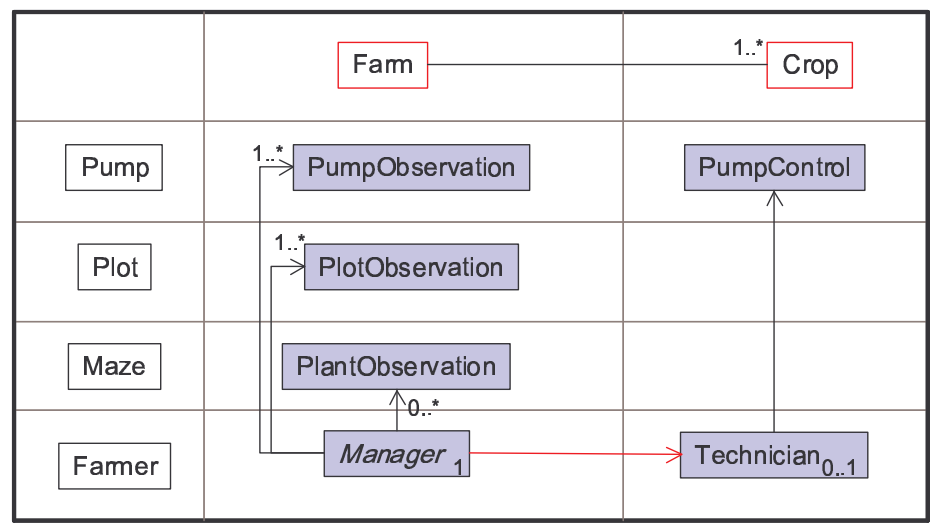

Figure 3. Diagramme swimlane de l'allocation de l'eau dans les exploitations

\subsubsection{Spécialisation des rôles du système}

Les diagrammes swimlane fournissent une représentation globale de la structure organisationnelle d'un système. Les rôles qui y sont définis peuvent se spécialiser pour définir différentes stratégies. On peut représenter ces différentes stratégies dans des diagrammes des classes UML. Ainsi dans le modèle-jouet, le rôle Manager se 
spécialise pour chacune des différentes phases de l'activité du gestionnaire de l'exploitation (semis, lancement l'irrigation, irrigation), puis pour définir des stratégies pour chacune de ces phases (figure 4)

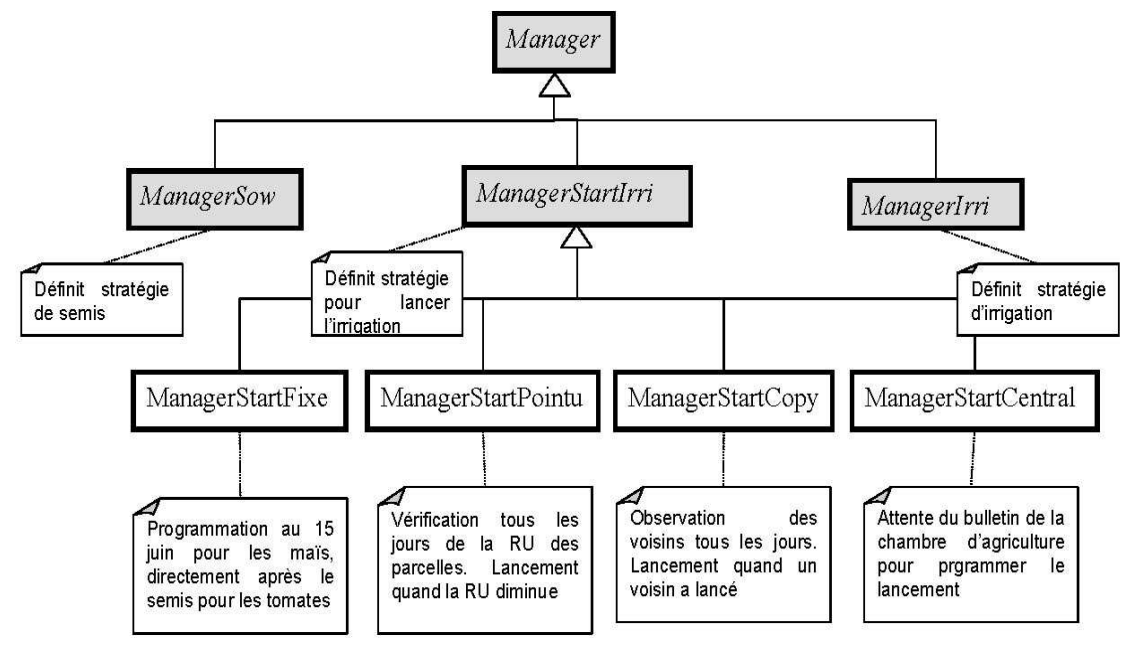

Figure 4. Diagrammes des classe du rôle Manager

\subsection{Identification des liens structurels entre les entités}

Enfin, il est possible d'utiliser les diagrammes des classes pour décrire les liens structurels existants entre les entités du système. Généralement, ces liens structurels sous-tendent les liens fonctionnels existant entre les rôles que prennent en charge ces entités. La figure 5 montre un extrait du diagramme structurel du modèle-jouet. Les liens fonctionnels sous-tendus sont notés par des contraintes.

\section{Application de la méthode ORIGAMI : description des dynamiques du système}

Il est maintenant temps de s'intéresser aux aspects dynamiques. Trois types de diagrammes dynamiques directement issus d'UML sont disponibles suivant que l'on souhaite décrire le déroulement d'un processus, d'une pratique ou d'une stratégie, le séquencement des interactions ou des dynamiques organisationnelles, ou bien le cycle de vie d'agents ou de rôles du système. 


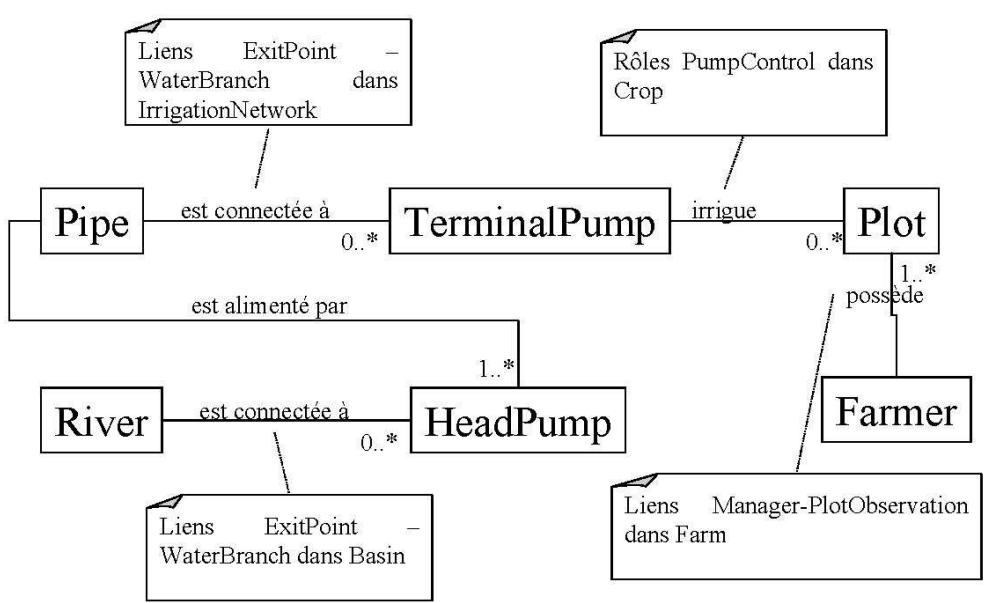

Figure 5. Diagramme structurel du modèle-jouet (extrait)

\subsection{Diagrammes d'activité}

Les diagrammes d'activité UML permettent classiquement de décrire la succession d'activité qu'une entité met en œuvre pour accomplir une certaine fonction ou un certain processus. Lorsque plusieurs entités sont impliquées dans le déroulement d'un processus, il est également possible d'utiliser des «swimlanes ». Nous proposons 3 types d'utilisation des diagrammes d'activité selon le niveau de détail voulu pour décrire l'accomplissement de processus ou de stratégie dans la représentation organisationnelle d'un système.

\subsubsection{Utilisation globale : articulations entre les niveaux d'organisation}

On peut utiliser les diagrammes d'activité de manière globale pour décrire les activités qui doivent s'enchaîner à travers les niveaux d'organisation lors de l'accomplissement d'un processus global du système. On ne représente alors que les groupes et les agents faisant le lien entre les groupes mis en jeu. Le diagramme d'activité global décrivant le rafraîchissement du débit dans le système est donnée figure 6. Il montre comment le débit se propage alors à travers les différents groupes physique. Les activités attribuées aux groupes doivent être mises en œuvre par les entités intervenant dans ces groupes à travers leurs rôles. 


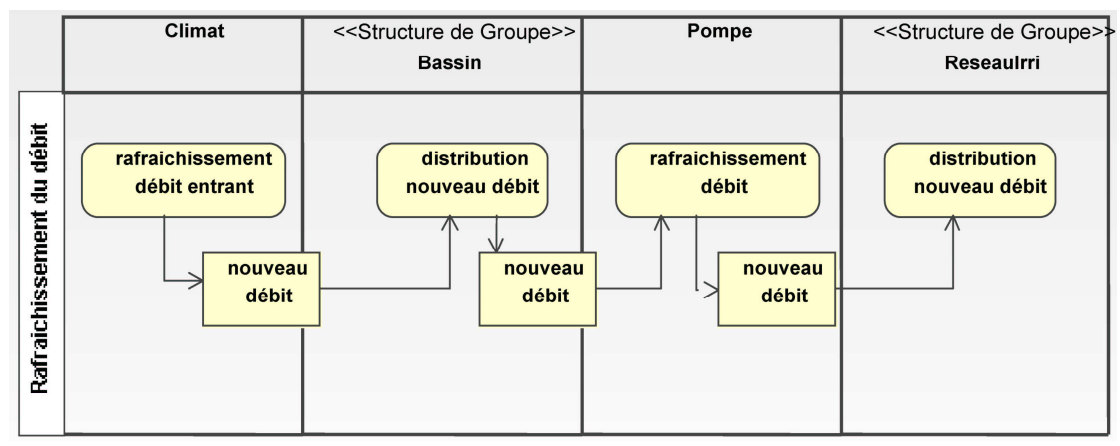

Figure 6. Diagramme d'activité global décrivant le rafraîchissement du débit dans le système

\subsubsection{Utilisation organisationnelle : activités au sein des niveaux d'organisation}

On peut aussi utiliser les diagrammes d'activité pour décrire les activités qui doivent s'enchaîner au travers des rôles définis dans un groupe pour en réaliser une activité globale. Des swimlanes sont alors utilisées pour représenter les différents rôles intervenant dans le groupe concerné. Le diagramme d'activité organisationnel décrivant la propagation du débit au sein d'un groupe Basin est donné figure 7 en illustration.

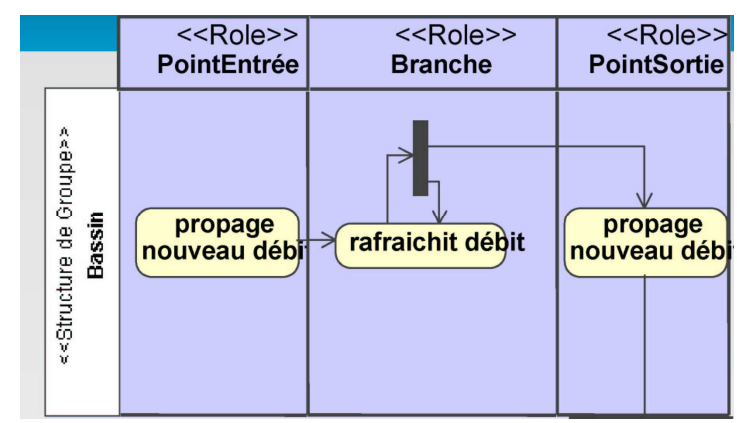

Figure 7. Diagramme d'activité organisationnel décrivant la propagation du débit au sein d'un groupe Basin

\subsubsection{Utilisation « classique » : description de pratiques ou de stratégies}

Enfin, les diagrammes d'activité peuvent être utilisés de manière plus classique, sans utilisation des swimlanes afin de décrire la succession des activités mises en 
œuvre par une entité (agent lui même ou un de ses rôles) pour mettre en œuvre une pratique ou une stratégie.

\subsection{Diagrammes de séquence}

Les diagrammes de séquence UML permettent de représenter le séquencement des interactions entre les entités d'un système. En général les diagrammes de séquence permettent une vue globale sur la succession des événements d'un modèle sans rentrer dans le détail de la description des dynamiques internes des entités (Le Page and Bommel 2005). (Ferber, Gutknecht et al., 2003) proposent une adaptation des diagrammes de séquences UML à la description de dynamiques organisationnelles (prises en charge et abandons de rôles) d'un système: les différents rôles définis au sein d'un même groupe sont définis au sein des mêmes cadres, alors que les différents rôles pris en charge par une même entités sont figurés par une même couleur; les prises en charge et abandons de rôles sont représentées par des créations et destructions de lignes de vie.

\subsection{Diagrammes d'état-transition}

Les diagrammes d'état-transition UML sont utilisés pour représenter le cycle de vie des entités d'un système par la définition des différents états qu'une entité peut rencontrer au cours d'une simulation, et surtout la définition des évènements provoquant la transition entre ces états. Ce type de diagramme est en général très utile pour décrire l'impact des pratiques des acteurs sur les objets du système. Dans le cadre de la représentation organisationnelle d'un système, les diagrammes d'étattransition peuvent aussi être utilisés pour décrire les rôles pris en charge et abandonnés par un agent durant son cycle de vie, ainsi que les transitions provoquant ces prises en charge. L'état d'un agent est alors décrit par l'ensemble des rôles qu'il prend en charge à un moment de la simulation. Cette utilisation des diagrammes d'état-transition est illustrée par la figure 8, qui représente la succession des rôles pris en charge par un agent Farmer.

Ce type de représentation permet de repérer quelles sont les prises en charge de rôles multiples dans le modèle. Cette prise en charge de rôles multiple doit se résoudre par des opérations de composition de rôles. La manière dont une composition de rôle s'effectue peut se décrire sur des diagrammes d'états-transition classiques, ou des conditions sur la présence ou l'action de rôles concurrents peuvent être notées en garde des transitions. 


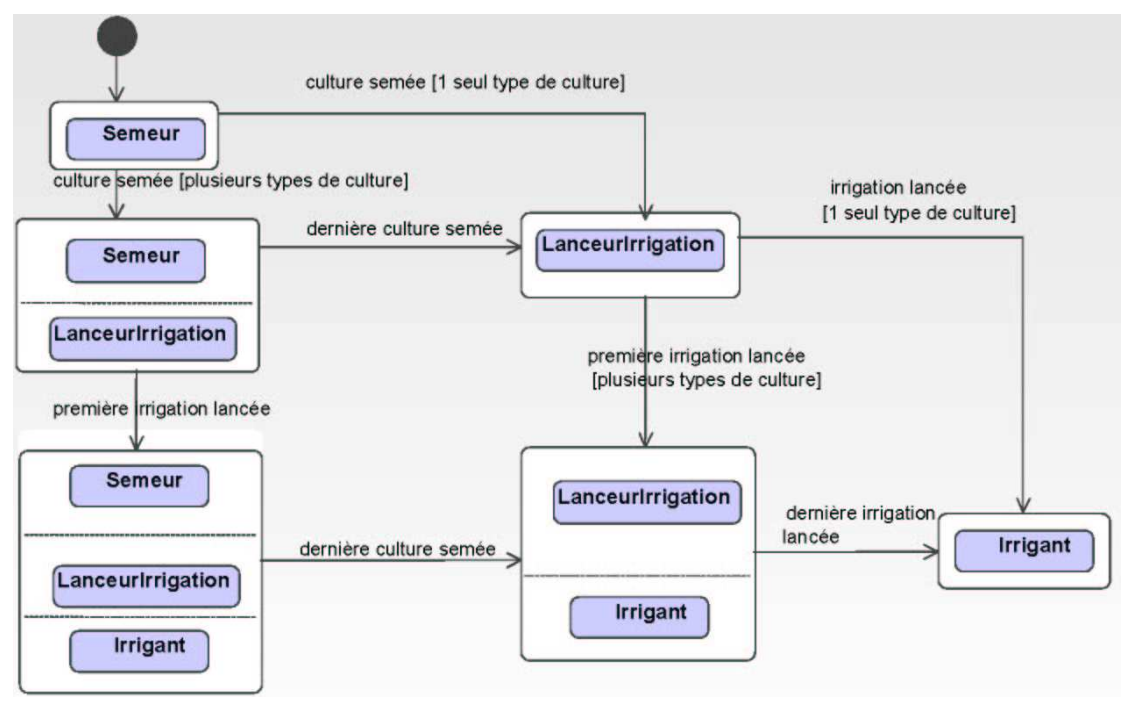

Figure 8. Diagramme d'état-transition de la succession des rôles d'un agriculteur

\section{Discussion}

La discussion se déroulera en 2 parties. Sur la base de l'exemple du modèlejouet d'abord, dans quelle mesure l'éventail des diagrammes proposés par ORIGAMI permet-il d'offrir des vues complémentaires sur la complexité d'un système? Puis sur la base de l'expérience d'une formation, dans quelle mesure l'approche est-elle portable et reproductible?

Mise en forme : Puces et numéros

\subsection{Gestion de la complexité par ORIGAMI}

Les apports d'ORIGAMI en termes de représentation de la complexité d'un système se situent principalement au niveau statique, puisque AGR permet de rendre compte de l'existence et de l'articulation de différents niveaux agrégatifs dans un système. En effet, au niveau statique, les diagrammes UML classiques permettent de structurer un système suivant 2 axes: liens conceptuels de spécialisation / généralisation entre les entités d'un système (diagramme des classes) ; relations concrètes (fonctionnelles ou structurelles) entre les catégories ou les entités d'un système (diagramme des classes ou diagramme d'objets). L'introduction de concepts organisationnels permet d'inclure une structuration et de dissocier le fonctionnel du structurel. Plus précisément :

- les diagrammes des classes permettent de décrire des hiérarchies d'entités aussi bien que des hiérarchies de rôles. L'existence des rôles donne donc accès à 
une unité de description généralisable de type fonctionnel plus petite et donc plus modulaire ;

- les diagrammes structurels et fonctionnels sont des diagrammes des classes sur lesquels on n'utilise que les associations entre classes. Le diagramme fonctionnel global, qui ne fait figurer que les groupes et leurs liens fonctionnels, offre une vue synthétique sur l'articulation entre les niveaux d'organisation d'un système. Le diagramme structurel, qui ne fait figurer que les agents et leurs liens structurels, offre une vue centrée exclusivement sur les relations intrinsèques et permanentes entre les entités du système ;

- le diagramme swimlane enfin constitue l'apport majeur d'ORIGAMI. Il représente dans un même diagramme les différents niveaux d'organisation et les différentes entités du système. Chacun des niveaux d'organisation est décrit par les rôles qu'il définit et leurs liens fonctionnels, et ces rôles sont placés en regard des entités susceptibles de les prendre en charge. Le diagramme swimlane offre une vue synthétique sur la structuration organisationnelle d'un système: une lecture verticale donne accès à la composition des niveaux d'organisation d'un système et à leur fonctionnement interne ; une lecture horizontale donne accès aux articulations entre ces niveaux d'organisation à travers la visualisations des rôles susceptibles d'être portés simultanément par un même agent.

Au niveau dynamique, ORIGAMI n'apporte pas de nouveau type de vue à UML. Elle ne fait qu'étendre les diagrammes dynamiques d'UML à la prise en compte d'entités organisationnelles.

Pour conclure, il faut souligner la pertinence du formalisme AGR, qui sous-tend la méthode ORIGAMI, vis-à-vis de la modélisation de systèmes socio-écologiques complexes. En effet celui-ci permet bien d'expliciter et représenter des niveaux d'organisation aussi bien physiques que sociaux, mais surtout d'exprimer leur articulation à travers la prise en charge de rôles par des entités du système. Non seulement il permet de structurer la description d'un système, mais il permet aussi d'en représenter les dynamiques : les dynamiques individuelles des entités prises en compte sont décrites pour les différents niveaux par les actions des rôles qu'elles prennent en charge, et les dynamiques globales émergent de l'interaction entre ces dynamiques individuelles.

Mise en forme : Puces et numéros

\subsection{Reproductibilité de la démarche}

A l'occasion d'une formation à la formalisation UML de systèmes complexes, le formalisme AGR et la méthodologie ORIGAMI ont été introduits aux participants en tant qu'abstractions et représentations complémentaires permettant une approche organisationnelle de leurs systèmes. Le public était composé de sociologues, de géographes, d'agronomes et de modélisateurs réunis autour de questions liées à l'affectation du foncier dans des systèmes comportant des échelles multiples. 
Durant la formation, les participants étaient organisés en 4 groupes travaillant chacun sur un système particulier. Ils devaient d'abord identifier les entités de leurs systèmes et leurs associations dans un diagramme des classes, puis effectuer une analyse organisationnelle afin d'en produire un diagramme swimlane. Chacun des groupes est parvenu à produire un tableau structure-fonction dont il a reconnu l'utilité pour classer les acteurs ou les pratiques de leur système suivant ses niveaux d'organisation. Seuls 2 des groupes ont eu le temps de produire des diagrammes swimlane. Les niveaux d'organisation ainsi identifiés étaient de nature différente suivant les groupes, ce qui démontre la capacité de l'approche à englober une diversité de points de vue :

- niveaux d'organisation induits par les échelles d'intervention des organisations sociales du système ;

- niveaux d'organisation induits par les pratiques existant autour des différents modes d'intervention sur le foncier (attribution des terres, mise en valeur des terres, conseil agricole) ;

- niveaux d'organisation induits par le placement dans la temporalité des processus de gestion: dynamiques naturelles (impact des règles de gestion), systèmes d'activité (mise en œuvre de règles de gestion) et processus de décisions (élaboration des règles de gestion). Puis selon les groupes redécoupage de ces niveaux suivant différents domaines ou objectifs (production, système économique, biodiversité, foncier...), ou bien suivant différentes échelles spatiales.

Le temps de la formation était trop court pour véritablement conclure sur la reproductibilité de la démarche. Cependant, l'expérience a montré la capacité de l'approche à apporter un mode de questionnement nouveau et à révéler des hypothèses implicites sur les systèmes: l'entrée organisationnelle a obligé les chercheurs a bien différencier les interactions liés à l'élaboration de règles, de celles liées à leur mise en œuvre, ou encore de celles liées à leur impact et à reconnaître les différentes échelles spatiales ou les différentes fonctions mises en jeu dans chacune de ces temporalités d'un processus de gestion. L'expérience a également démontré la capacité du formalisme AGR à fournir des éléments de description génériques. Enfin l'expérience a souligné la nécessité de mieux clarifier les concepts de niveaux d'organisation et de rôle, trop fortement polysémiques et ambigus pour un public composé de chercheurs issus de disciplines différentes.

Mise en forme : Puces et numéros

\section{Conclusion}

La méthodologie ORIGAMI fournit des diagrammes qui permettent de construire une représentation organisationnelle d'un système. Ces diagrammes apportent des points de vue complémentaires à ceux offerts par une approche UML « classique ». Parmi ces diagrammes, le diagramme swimlane est ressorti comme particulièrement fécond pour discuter d'un modèle ou dégrossir l'analyse d'un système par le point de vue structurant qu'il amène. 
Le formalisme AGR, qui sous-tend la méthodologie ORIGAMI permet de répondre à certains écueils de la modélisation multi-agent classique en fournissant les abstractions organisationnelles de rôles et de groupes. Les rôles permettent de faire des fonctions identifiées dans un système des composants modulaires et ainsi de résoudre des problèmes liés à la confusion entre identité, fonctions et pratiques d'une entité. Les groupes permettent de faire des niveaux d'organisation d'un système des objets manipulables et ainsi de définir des sous-systèmes cohérents plus propices à être généralisés.

La décomposition du système en groupes et en rôles, et l'obligation de toujours exprimer la participation d'un agent dans un groupe par un rôle et de restreindre les interactions entre agents aux interactions via des rôles pris en charge dans un même groupe a cependant pour conséquence de multiplier le nombre de classes à définir pour développer un modèle. Ainsi certains rôles ne sont créés que par la nécessité d'exprimer la participation d'un agent à un groupe. On peut cependant toujours penser que l'introduction d'un nouveau rôle n'est jamais inutile, et permet d'expliciter et de formaliser la participation d'une entité à un niveau d'organisation.

Cette multiplication d'objets à définir, qui représente une complexité initiale accrue pour le modélisateur est sans doute le prix à payer pour forcer à l'explicitation d'hypothèses sur les modes d'actions des agents dans les différents niveaux d'organisation. Le choix d'utiliser un formalisme structuré tel qu'AGR repose donc sur un équilibre entre exigence de simplicité et exigence d'explicitation des hypothèses sur un système.

\section{Remerciements}

Nous remercions les participants à la formation UML-AGR qui s'est tenu au Cirad en juin 2005 de s'être essayé à l'utilisation d'ORIGAMI et d'avoir ainsi contribué à la clarification des concepts et de la méthodologie.

\section{Bibliographie}

Abrami, G., O. Barreteau, F. Cernesson et S. Lardon (2003). Implementing the Agent-GroupRole architecture in an irrigation water management application. 4th workshop on agentbased simulation, Montpellier, France, SCS.

Barreteau, O., P. Garin, Dumontier, A., Abrami, G. et Cernesson, F., (2003). "Agent based facilitation of water allocation : case study in Drome River Valley." Group Decision and Negotiation 12(5): 441-461.

Bousquet, F. (2001). Modélisation d'accompagnement et gestion des ressources renouvelables, Université Lyon 1: 70 p. 
Bousquet, F., O. Barreteau, d'Aquino, P., Etienne, M., Boissau, S., Aubert, S., Le Page, C., Babin, D. et Castella, J.-C (2002). Multi-agent systems and role games : collective learning processes for ecosystem management. Complexity and ecosystem management. M. A. Janssen, Edward Elgar.

Bousquet, F. et C. Le Page (2004). "Multi-agent simulations and ecosystem management: a review." Ecological Modelling 176(3-4): 313-332.

Drogoul, A., D. Vanbergue, et Meurisse T. (2003). Multi-agent Based Simulation: Where Are the Agents?, MABS 2002.

Ferber, J. and O. Gutknecht (1998). A meta-model for the analysis and design of organizations in multi-agents systems. ICMAS'98, Paris, IEEE Computer Society.

Ferber, J., O. Gutknecht et Michel F.. (2003). Agent/Group/Roles: Simulating with organization. 4th workshop on agent-based simulation, Montpellier, France, SCS.

Heathcote, I. W. (1998). Integrated watershed management, principles and practice, John Wiley $\backslash \&$ Sons.

Heemskerk, M., K. Wilson, et Pavao-Zuckerman, M. (2003). "Conceptual Models as Tools for Communication Across Disciplines." Conservation Ecology [online] 7(3): $\operatorname{url}\{\mathrm{http}: / /$ www.consecol.org/vol7/iss3/art8\}.

Heylighen, F. (1999). "Advantages and limitations of formal expression." Foundations of Science 4(1): 25-56.

Le Page, C. and P. Bommel (2005). The CORMAS simulation platform, a toolkit designed for using multi-agent systems in the field of integrated natural resources management. Companion modeling and multi-agent systems for integrated natural resources management in Asia. F. Bousquet, G. Trébuil and B. Hardy. Los Baños, Philippines, International Rice Research Institute.

Parunak, H. V. D. and J. Odell (2002). Representing social structures in $\{U M L\}$. AgentOriented Software Engineering II, Montreal, Canada, Springer.

Roux-Rouquie, M., N. Caritey, Gaubert, L. and Rosenthal-Sabroux, C.,. (2004). "Using the Unified Modelling Language (UML) to guide the systemic description of biological processes and systems." Biosystems 75(1-3): 3-14.

Zanker, S. (1999). Modél isation et gestion de la demande agricole en eau dans la Drôme, cas de la mise en application du SAGE Drôme, CEMAGREF: 55. 